CLINICAL STUDY

\title{
Frequent incidental discovery of phaeochromocytoma: data from a German cohort of 201 phaeochromocytoma
}

\author{
Robert Kopetschke, Mario Slisko ${ }^{1}$, Aylin Kilisli, Ulrich Tuschy ${ }^{2}$, Henri Wallaschofski ${ }^{3}$, Martin Fassnacht ${ }^{4}$, \\ Manfred Ventz, Felix Beuschlein ${ }^{1}$, Martin Reincke ${ }^{1}$, Nicole Reisch ${ }^{1}$ and Marcus Quinkler \\ Clinical Endocrinology, Charité Campus Mitte, Charité University Medicine Berlin, Charitéplatz 1, D 10117 Berlin, Germany, ${ }^{1}$ Medizinische Klinik \\ Innenstadt, Ludwig-Maximilians-Universität München, München, Germany, ${ }^{2}$ Bereich Endokrinologie, 2. Medizinische Klinik, Helios Klinikum Erfurt, \\ Erfurt, Germany, ${ }^{3}$ Institut of Clinical Chemistry and Laboratory Medicine, Ernst-Moritz-Arndt-Universität, Greifswald, Germany and ${ }^{4}$ Endocrinology and \\ Diabetes Unit, Department of Medicine I, University Hospital, University of Wuerzburg, Wuerzburg, Germany
}

(Correspondence should be addressed to M Quinkler; Email: marcus.quinkler@charite.de)

\begin{abstract}
Context: Adrenal and extra-adrenal phaeochromocytoma are chromaffin cell-derived tumours that are discovered due to classical symptom triad with headache, sweating and palpitations combined with persistent or paroxysmal hypertension. However, an increasing proportion of phaeochromocytoma seems to be discovered incidentally upon abdominal imaging.

Objective: To specify the exact circumstances of discovery of adrenal and extra-adrenal phaeochromocytoma.

Design and patients: Four German endocrine centres participated in this retrospective study. Medical records of 201 patients with adrenal and extra-adrenal phaeochromocytoma who were diagnosed between 1973 and 2007 were analyzed.

Results: The typical triad of symptoms was found only in $10 \%$ of cases. Ten percent of patients presented were without clinical symptoms and $6.1 \%$ were normotensive. Documented blood pressure peaks occurred in $44.1 \%$ of cases. In 24 patients $(12.2 \%)$, phaeochromocytoma was malignant. Before $1985,<10 \%$ of cases were incidentally discovered, whereas thereafter the frequency was $>25 \%$ (29.4\% of the total study population). Patients with incidentally detected phaeochromocytoma were significantly older $(53.1 \pm 1.9$ vs $47.0 \pm 1.3$ years; $P<0.05)$ and often had less blood pressure peaks $(37.0$ vs $70.7 \% ; P<0.001)$ than patients in whom the diagnosis was suspected on clinical grounds. Of phaeochromocytomas $94.4 \%$ were intra-adrenal tumours, of which $12.9 \%$ were bilateral. Bilateral tumours were significantly smaller than unilateral tumours $(36.6 \pm 14.7$ vs $52.5 \pm 34.3 \mathrm{~mm} ; P<0.05)$, whereas extra-adrenal tumours had a mean diameter of $52.6 \pm 28.7 \mathrm{~mm}$.

Conclusions: Owing to better availability and accessibility of imaging procedures, the number of incidentally discovered phaeochromocytoma is increasing and reaches nearly $30 \%$ in our study population. Every adrenal incidentaloma should be investigated for the presence of phaeochromocytoma.
\end{abstract}

European Journal of Endocrinology 161 355-361

\section{Introduction}

The nomenclature of chromaffin cell tumours is not generally accepted. According to the WHO 2004 classification (1), there are two types of neoplasm's derived from chromaffine tissue: phaeochromocytomas (tumours of adrenal medulla); and paragangliomas (tumours located extra-adrenally). However, in several publications these tumours are divided into cataecholamine-producing tumours that are derived from the sympathetic neuroendocrine system (adrenal and extraadrenal phaeochromocytomas) and in tumours derived from the parasympathetic system. The latter tumours are classified as paragangliomas and are usually not cataecholamine-secreting. In this manuscript, we are following the latter nomenclature using the terms adrenal and extra-adrenal phaeochromocytomas.

Phaeochromocytomas are rare with an incidence of 1-6 per million and a prevalence of $0.1-0.6 \%$ in patients with hypertension. However, an Australian autopsy series revealed a prevalence of $0.05 \%$ in this unselected population (2) suggesting that many tumours are overseen in clinical practice. Phaeochromocytoma may occur sporadically or as part of a hereditary syndrome. They synthesise the cataecholamines noradrenaline, adrenaline and more rarely dopamine (3), either isolated or in combination. Phaeochromocytoma can be fatal and it is important 
to diagnose the disease while it is still curable by surgery. Because the tumours are most often benign, surgical resection usually provides an effective treatment and cure.

Owing to the unpredictable, often acute nature of cataecholamine secretion, the tumours can be present with a host of symptoms and variable, often confusing, clinical manifestations (4). Headache, palpitations and sweating are described as the most common symptoms (5); however, several studies showed a significant delay of the diagnosis phaeochromocytoma in many patients $(6,7)$. The rarity and variability render these tumours very difficult to diagnose. The laboratory diagnosis of these tumours is based on the identification of excessive secretion of cataecholamines and/or of their derivates $(8,9)$. Since 1998, assays of plasma fractionated metanephrines have been developed and used progressively thereafter $(10,11)$.

A recent report of 41 patients suggested that nowadays many phaeochromocytomas are diagnosed incidentally during radiological examinations of the abdomen or at autopsy (12). Thus, the purpose of this retrospective study was to determine the frequency of incidental discovery of adrenal and extra-adrenal phaeochromocytoma in 201 consecutive cases at four German endocrine centres between 1973 and 2007.

\section{Methods}

\section{Subjects}

We have retrospectively analyzed the files of all patients who were diagnosed with adrenal or extra-adrenal phaeochromocytoma at four German endocrine centres between 1973 and 2007. A total of 101 patients were diagnosed before the year 2000 with a subset of 29 patients before the year 1990. The study was approved by the ethical committee of the Charité Campus Mitte Berlin (permit no. EA1/136/08). The only inclusion criterion was a past or new diagnosis of adrenal or extra-adrenal phaeochromocytoma. Patients with parasympathic paragangliomas were excluded. The cases were identified from the files of the participating departments by trained medical students using a structured evaluation form. For the purpose of this study, medical records on the first consultation were analyzed to determine how the diagnosis was established. Detailed personal and family history, the referred signs and symptoms, physical examination, systolic and diastolic blood pressure and heart rate, biochemical analysis and imaging were also reviewed at the time the tumour was initially detected. In addition, surgical procedures, perioperative complications and pathology reports were evaluated. In 109 patients, clinical data during the follow-up could be retrieved (median followup 10.7 months; range 0.5-432 months). Systolic and diastolic blood pressures of $\geq 140$ and $\geq 90 \mathrm{mmHg}$ respectively, were considered to be increased. Hypertension was defined as increased systolic or diastolic blood pressure or use of antihypertensive medication. A hypertensive peak was defined as a sudden elevation in systolic blood pressure by $>50 \%$ of the preceding measurement. Genetic counselling differed between centres. Whereas in the Munich centre, all subjects received routine genetic screening for mutations for MEN2, von Hippel-Lindau syndrome (VHL) etc., centres in Berlin, Erfurt and Greifswald performed genetic testing only in individual cases. Data were collected using an Excel electronic spreadsheet.

\section{Statistical analysis}

The mean and s.D. were calculated for each numeric variable. Significance of differences was determined by unpaired $t$-test, Mann-Whitney-Wilcoxon test or Fisher's exact test where appropriate. Significance was accepted if $P<0.05$. Analyses were performed by using the statistical software package SPSS, version 13.0 (SPSS Inc., Chicago, IL, USA).

\section{Results}

\section{Study population}

The patients were aged between 12 and 85 years. About $50.7 \%$ of the patients were females. There was no significant difference between the sexes with regard to age, body mass index or blood pressure at the time of diagnosis. The mean time interval from the first onset of symptoms until final diagnosis of phaeochromocytoma was $3.9 \pm 5.2$ years, with a median of 1.7 years (range: 0.6-24.3 years). Table 1 reports clinical symptoms and signs of patients at the time of diagnosis. About 32.4\% of the patients did not display any signs of the triad, whereas $10.1 \%$ of them had no symptoms at all.

Table 1 Percentage of symptoms and clinical signs in 183 patients who were diagnosed with phaeochromocytoma between 1973 and 2007 in four German centres.

\begin{tabular}{lc}
\hline Variables & Percentage (\%) \\
\hline Sweating & 46.9 \\
Headaches & 36.3 \\
Palpitations & 34.1 \\
Loss of weight & 17.3 \\
Vertigo & 16.8 \\
Asthenia & 16.2 \\
Anxiety/uneasiness/insomnia & 15.1 \\
Nausea/vomiting & 14.5 \\
Tremor & 11.2 \\
Headaches + palpitations + sweating & 10.1 \\
$\quad$ (complete triad) & \\
Paleness & 9.5 \\
Lower abdominal pain & 8.4 \\
Upper abdominal pain & 6.7 \\
Angina pectoris & 2.8 \\
Absence of symptoms & 10.1 \\
\hline
\end{tabular}


Of the patients, 93.9\% had documented hypertension, whereas in $6.1 \%$ hypertension was not detectable. Of the hypertensive patients, $8.2 \%$ presented only with blood pressure peaks and had no evidence for permanent hypertension. Of the patients, $35.9 \%$ had persistent hypertension with blood pressure peaks and $55.9 \%$ without blood pressure peaks. Before establishment of the diagnosis, $70.6 \%$ of the hypertensive patients were treated with anti-hypertensive drugs, mostly $\beta$-blockers (65.2\%), ACE-inhibitors (40.0\%), calcium channel blockers (33.0\%) and $\alpha$-blockers $(20.9 \%)$. The average number of anti-hypertensive medication classes was $1.4 \pm 1.2$.

The results of preoperative hormonal assays are given in Table 2. Owing to the diversity of hormonal assays used over the last 35 years and due to differences in normal range levels, we analyzed our cohort only with regards to normal or pathologically elevated results in the hormonal assays. All patients had at least one abnormal hormonal parameter.

\section{Incidental finding}

The different means of detecting phaeochromocytoma are summarised in Table 3. High blood pressure, blood pressure peaks and other typical symptoms led to the discovery of phaeochromocytoma in $70.6 \%$ of the cases, whereas 29.4\% were found incidentally. Among patients who were identified with phaeochromocytoma incidentally by abdominal imaging, $87.5 \%$ were hypertensive. Table 4 shows the comparison of patient's characteristics and tumour data according to the circumstances of detection of the phaeochromocytoma. Interestingly, patients with incidentally detected tumours were significantly older, and presented less often hypertension and blood pressure peaks than patients with clinically suspected phaeochromocytoma.

Table 2 Preoperative hormonal data of 201 cases that were diagnosed with adrenal or extra-adrenal phaeochromocytoma between 1973 and 2007 in four German centres. Owing to different hormonal assays used and owing to different ranges of normal values, no absolute hormone levels were shown.

\begin{tabular}{lc}
\hline Variables & $\begin{array}{c}\text { Pathological } \\
\text { finding/total tested (\%) }\end{array}$ \\
\hline Plasma adrenaline & $7 / 8(87.5)$ \\
Plasma noradrenaline & $8 / 8(100)$ \\
Plasma dopamine & $1 / 5(20)$ \\
Plasma metanephrine & $5 / 6(83.3)$ \\
Plasma normetanephrine & $5 / 6(83.3)$ \\
Urinary metanephrines & $44 / 69(63.8)$ \\
Urinary normetanephrines & $53 / 63(84.1)$ \\
Urinary adrenaline & $104 / 163(63.8)$ \\
Urinary noradrenaline & $130 / 164(79.3)$ \\
Urinary dopamine & $42 / 139(30.2)$ \\
Urinary VMA & $71 / 86(82.6)$ \\
Chromogranin A & $4 / 13(30.8)$ \\
NSE & $7 / 25(28.0)$ \\
\hline
\end{tabular}

VMA, vanillylmandelic acid and NSE, neuron-specific enola.
Table 3 Circumstances of discovery of 201 cases that were diagnosed with adrenal or extra-adrenal phaeochromocytoma between 1973 and 2007 in four German centres.

\begin{tabular}{lc}
\hline & Percentage (\%) \\
\hline $\begin{array}{l}\text { Suspected finding (hypertension+ } \\
\text { typical symptoms) }\end{array}$ & 70.6 \\
Incidental finding (due to imaging for:) & 29.4 \\
Routine examination & 7.0 \\
Suspected tumour or malignancy, & 5.0 \\
e.g. decreased physical activity & \\
Suspected renal abnormality, & 3.0 \\
$\quad$ microhematuria & \\
Undefined abdominal pain, e.g. & 3.5 \\
$\quad$ tenesmen, meteorism, gallbladder & \\
stones & \\
Paravertebral/vertebral pain & 3.0 \\
Follow up other disease, & 2.5 \\
$\quad$ e.g. M Crohn & \\
Acute inflammatory disease & 2.5 \\
Ulcus ventriculi/duodeni & 1.5 \\
Operative finding & 0.5 \\
Decompensated diabetic mellitus & 0.5 \\
Anaemia & 0.5 \\
\hline
\end{tabular}

Figure 1 shows the rate of incidental finding in three subperiods (each one about 11 years) demonstrating that the frequency of incidentally detected tumours was much lower at the time before modern imaging techniques (ultrasound, computerised tomography (CT), magnetic resonance imaging (MRI)) were widely available.

\section{Imaging}

In $85 \%$ of the patients, CT scan was performed. Of the patients, $32.6 \%$ received a preoperative MRI. Total body scan by metaiodobenzylguanidine was done in $74.3 \%$ of the patients, and an octreoscan in $3.2 \%$. Of the patients, $23 \%$ received one imaging examination, $62 \%$ of the patients received two imaging techniques, and $15 \%$ of patients received three different imaging examinations.

A total of 186 tumours were intra-adrenal, out of which $87.1 \%$ were unilateral and $12.9 \%$ were bilateral. Of the unilateral tumours (largest diameter: mean \pm s.D.; $52.5 \pm 34.3 \mathrm{~mm}$ ), $51.9 \%$ were located in the right adrenal and $48.1 \%$ in the left adrenal gland. Right-sided intraadrenal tumours were $52.7 \pm 27.5 \mathrm{~mm}$ in size, and leftsided intra-adrenal tumours were $52.5 \pm 40.6 \mathrm{~mm}$ in size. Bilateral tumours $(36.6 \pm 14.7 \mathrm{~mm})$ were significantly smaller $(P<0.05)$ than unilateral tumours, whereas the extra-adrenal tumours had a mean diameter of 52.6 $\pm 28.7 \mathrm{~mm}$. Extra-adrenal localisations were paraaortal and retrocaval in the aortic bifurcation, retroperitoneal, in the left kidney, the bladder or in the vagina.

\section{Surgery}

Data from 174 patients could be analyzed regarding the surgical approach: 96 patients $(55.2 \%)$ were operated by laparotomy, whereas 78 patients $(44.8 \%)$ underwent 
Table 4 Patient's characteristics according to the circumstances of discovery in 201 adrenal or extra-adrenal phaeochromocytoma.

\begin{tabular}{llll}
\hline Variables & $\begin{array}{l}\text { Incidental finding } \\
(n=59)\end{array}$ & $\begin{array}{l}\text { Suspected finding } \\
(n=142)\end{array}$ & $\begin{array}{l}\text { Level of } \\
\text { significance }\end{array}$ \\
\hline Gender (women/men) & $30 / 29$ & $72 / 70$ & $P<0.05$ \\
Age at diagnosis (years) & $53.1 \pm 1.9$ & $47.0 \pm 1.3$ & $\mathrm{NS}$ \\
BMl $\left(\mathrm{kg} / \mathrm{m}^{2}\right)$ & $24.6 \pm 0.7$ & $26.1 \pm 0.7$ & $\mathrm{NS}$ \\
Systolic BP (mmHg) & $148 \pm 4$ & $152 \pm 3$ & $\mathrm{NS}$ \\
Diastolic BP (mmHg) & $89 \pm 2$ & $92 \pm 2$ & $\mathrm{NS}$ \\
Mean arterial BP (mmH) & $109 \pm 2$ & $112 \pm 2$ & $\mathrm{NS}$ \\
Heart rate (b.p.m.) & $81 \pm 3$ & $80 \pm 2$ & $P<0.01$ \\
Prevalence of headaches + palpitations + sweating & $3.4 \%$ & $\mathrm{NS}$ \\
Prevalence of hypertension & $87.5 \%$ & $96.8 \%$ & $P<0.001$ \\
Prevalence of consistent blood pressure & $87.0 \%$ & $89.3 \%$ & $\mathrm{NS}$ \\
Prevalence of blood pressure peaks & $37.0 \%$ & $70.7 \%$ & \\
Tumour diameter (mm) & $60.0 \pm 7.5$ & $47.3 \pm 2.2$ & \\
\hline
\end{tabular}

Means \pm S.D. BP, blood pressure and BMI, body mass index.

a laparoscopic approach. In seven patients, the laparoscopic operation was converted into laparotomy (9\%). There was no documented mortality. Intraoperative blood pressure peaks were documented in nine cases $(5.2 \%)$, hypotensive phases in five cases $(2.9 \%)$ and one cardiovascular crisis occurred $(0.6 \%)$.

\section{Blood pressure changes after surgery}

The effects of tumour removal on blood pressure could be evaluated in 109 patients. Mean systolic as well as the diastolic blood pressure decreased significantly (from $155 \pm 32$ to $132 \pm 19 \mathrm{mmHg}$; and from $94 \pm 17$ to $80 \pm 14 \mathrm{mmHg}$ respectively; both $P<0.001)$. The mean arterial blood pressure decreased significantly $(P<0.001)$ from $114 \pm 21 \mathrm{mmHg}$, before surgery to $97 \pm 14 \mathrm{mmHg}$ at the first follow-up visit after surgery. Before operation, $94.5 \%$ of the 109 patients were hypertensive. After operation, hypertension persisted in $42 \%$ of these patients. The number of antihypertensive medication classes was significantly reduced after operation (mean \pm s.E.M.; $1.4 \pm 0.1$ vs $0.9 \pm 0.1$; $P<0.005)$.

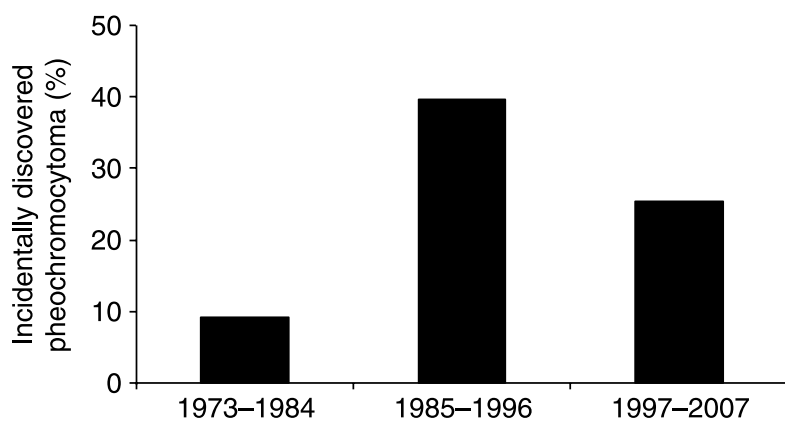

Figure 1 Percent of incidentally discovered phaeochromocytomas depending on subperiods (1973-1984, $n=11$; 1985-1996, $n=68$; 1997-2007, $n=122)$. In 1985, ultrasound machines became widely available in general practice in Germany and spiral CT was introduced in 1989.

\section{Malignancy}

A total of 24 phaeochromocytomas (12.2\%) were classified as malignant at diagnosis (18 adrenal unilateral tumours and six extra-adrenal tumours). A total of 22 out of the 24 malignant tumours were diagnosed by evidence of metastases in lymph nodes $(n=9)$, liver $(n=7)$, lung $(n=3)$ and bone $(n=3)$. The other two tumours were classified malignant due to the histological finding, such as capsule invasion and blood vessel infiltration. Unfortunately, no follow-up data were available for these two patients. Extra-adrenal tumours $(n=11)$ were significantly $(P<0.001)$ more often malignant (54.6\%) than intra-adrenal tumours (9.7\%).

\section{Genetic testing}

In 10 patients of our study population, a hereditary syndrome was diagnosed (two patients with VHL; seven patients with MEN2; one patient with neurofibromatosis type 1 (NF1)). In addition, only 73 out of 201 patients with adrenal and extra-adrenal phaeochromocytoma $(36.3 \%)$ received genetic testing (in total 253 genetic examinations). In this group, $20(27.4 \%)$ patients showed genetic mutations (four patients with VHL, 10 patients with MEN2A, five patients with NF1, one patient with succinate dehydrogenase complex subunit D (SDHD)). Only 9 out of the 24 malignant phaeochromocytomas were genetically tested; all of them were negative for $S D H B, S D H C$ and $S D H D$ mutations.

\section{Discussion}

The present retrospective study confirms the wide variation of the clinical spectrum in patients with phaeochromocytoma and that classical symptoms are far more infrequent; the complete classical triad occurred in about $10 \%$ of patients compared with $15-24 \%$ in other series (12-14). In our study, 
approximately half of the patients had permanent hypertension, and $44.1 \%$ of patients suffered from blood pressure peaks compared with $67.1 \%$ in an Italian study (13). Interestingly, $6.1 \%$ of our phaeochromocytoma patients were normotensive, emphasising that the absence of hypertension does not rule out the presence of a cataecholamine-producing tumour that might result in life-threatening situations (15). One explanation may be that tumour-dependent hormone production may remain silent for a long period of time, because active cataecholamines can be converted into biologically inactive metanephrines by cataechol-Omethyltransferase within the tumour $(8,16,17)$. Owing to the rare occurrence, the often unspecific signs and symptoms, and the low sensitivity of any symptoms and the diagnosis of phaeochromocytoma may be delayed. This difficulty explains the long time lag (mean: $3.9 \pm 5.9$ years) between initial symptoms and diagnosis in our patient's cohort. In addition, this is reflected also by the high rate (nearly 30\%) of incidental finding in our study. In two recent studies, 19 out of 33 patients $(57.6 \%)(18)$ and 19 out of 46 patients $(41 \%)$ (19) with adrenal phaeochromocytoma had tumours discovered incidentally on imaging, however, these were rather small cohorts. Another small study with 41 phaeochromocytoma reported a finding in $51 \%$ of cases due to blood pressure abnormalities, in 29\% imaging due to pain examinations and in $20 \%$ of cases due to various pathologies (12). However, they were included in the latter group patients with known MEN2A and neurofibromatosis, resulting in a questionable definition of incidental finding. Other studies reported the lower percentages (10-15\%) of incidental findings $(13,20$, 21). Our series demonstrates that before the widespread distribution of ultrasound screening in general practice and before the introduction of $\mathrm{CT}$ scans into clinical routine, the rate of incidentally discovered phaeochromocytomas was $<10 \%$. We assume that the rate of incidentally discovered phaeochromocytomas considerably increased due to, first, the increased availability and more frequent use of MRI, CT or abdominal ultrasound and secondly, the better overall health education and awareness of the population and improved accessibility of general practitioners, internists and endocrinologists.

With widespread application of abdominal imaging, an increasing number of adrenal masses are being found incidentally (up to $3.4 \%$ of all abdominal CT scans) (22), and autopsy studies have shown a prevalence of $8 \%$ (23). Several clinical studies revealed that $\sim 5-7 \%$ of all incidentaloma of the adrenal gland are phaeochromocytoma (24-27). Therefore, it is necessary to thoroughly evaluate all patients with an isolated adrenal mass.

Incidentally discovered phaeochromocytomas are diagnosed in many patients before any symptoms develop. The prevalence of an asymptomatic phaeochromocytoma is estimated to be $11-21 \%(4,13,21)$, and retrospective studies have failed to define difference in demographic, radiographic and pathological characteristics in sporadic tumours $(22,28)$. In accordance with another study (21), patients with incidentally discovered phaeochromocytoma in our study population were significantly older than the patients with findings indicative of cataecholamine excess. We found a trend towards larger phaeochromocytomas in patients with incidental finding. By contrast, the study by Amar et al. (21) did not find a difference in tumour size between incidentally and suspected finding, but showed that patients with incidentaloma had lower plasma cataecholamine concentrations than patients with adrenergic symptoms.

In our study, there was no difference between the occurrence in the right and left adrenal; however, other phaeochromocytoma series reported a significantly more frequent occurrence in the right adrenal (13, 21 ), but could not provide a sound explanation for their finding. Furthermore, in our series only $7.5 \%$ of the phaeochromocytoma had an extra-adrenal localisation. In a Swedish study, 22\% of the detected phaeochromocytoma were located outside the adrenals; however, the series also included diagnosis achieved by autopsy (20). Other studies (13) showed extra-adrenal location in $8.5 \%$ of phaeochromocytoma supporting our data. The percentage of malignant phaeochromocytoma was in the same range as reported by most other series $(4,9,21)$.

In our study, far less intraoperative complications occurred $(8.7 \%)$ than previously reported (4). Other studies reported up to $15 \%$ intra- or perioperative complications $(12,29)$. In our series, $9 \%$ of laparoscopic operations had to be converted into an open approach, which might be due to the introduction of laparoscopic adrenalectomy during our study period. Nowadays, laparoscopic adrenalectomy is regarded as safe and to be the operation of choice with very few complications $(30,31)$.

Our retrospective study has the limitations that the diagnostic (especially hormonal assays) and follow-up approach was different among the centres and during the studied period, and that a large number of patients was lost in the follow-up. In addition, a lack of appropriate genetic screening in all centres is evident in our study. It must be stressed, however, that genetic screening for this tumour entity has become standard of care not before 2002. All participating centres were endocrine units that might have kept the rate of incidentally detected phaeochromocytoma in a lower range. It is conceivable that in surgical or urological series the incidentally detected rate is even higher. In addition, most of the centres were located in Northern Germany and only one centre in Southern Germany adding a potential bias to our study due to the fact that hypertension is more prevalent in the Northern German population (32). 
In conclusion, our study confirms the great variability of the clinical picture of phaeochromocytoma. Most probably due to improvements in availability and accessibility of imaging techniques, the number of incidentally discovered phaeochromocytoma is increasing, further indicating the necessity for sufficient screening for cataecholamine excess in all patients with adrenal incidentaloma.

\section{Declaration of interest}

The authors declare that there is no conflict of interest that could be perceived as prejudicing the impartiality of the research reported.

\section{Funding}

This research did not receive any specific grant from any funding agency in the public, commercial or not-for-profit sector.

\section{Acknowledgements}

We are very thankful to Prof. Dr Wolfgang Oelkers, Endokrinologikum Berlin, for help with identification of phaeochromocytoma patients. We are also grateful to Prof. Dr Hartmut Neumann, University of Freiburg, for genetic analysis in a subset of patients. This work was part of an initiative of the German Adrenal Network GANIMED.

\section{References}

1 De Lellis RA, Lloyd RA, Heitz PU \& Eng C. Pathology and Molecular Genetics of Endocrine Tumours. WHO Classification of Tumours of Endocrine Organs Lyon: IARC Press, 2004.

2 McNeil AR, Blok BH, Koelmeyer TD, Burke MP \& Hilton JM. Phaeochromocytomas discovered during coronial autopsies in Sydney, Melbourne and Auckland. Australian and New Zealand Journal of Medicine 200030 648-652.

3 Dubois LA \& Gray DK. Dopamine-secreting pheochromocytomas: in search of a syndrome. World Journal of Surgery 200529 909-913.

4 Goldstein RE, O'Neill JA Jr, Holcomb GW, Morgan WM, Neblett WW, Oates JA, Brown N, Nadeau J, Smith B, Page DL, Abumrad NN \& Scott HW Jr. Clinical experience over 48 years with pheochromocytoma. Annals of Surgery 1999229 755-764.

5 Manger WM \& Eisenhofer G. Pheochromocytoma: diagnosis and management update. Current Hypertension Reports 20046 477-484.

6 Kizer JR, Koniaris LS, Edelman JD \& St John Sutton MG. Pheochromocytoma crisis, cardiomyopathy, and hemodynamic collapse. Chest 2000118 1221-1223.

7 Schwarz RJ \& Schmidt N. Efficient management of adrenal tumors. American Journal of Surgery $1991 \mathbf{1 6 1} 576-579$.

8 Baguet JP, Hammer L, Mazzuco TL, Chabre O, Mallion JM, Sturm N \& Chaffanjon P. Circumstances of discovery of phaeochromocytoma: a retrospective study of 41 consecutive patients. European Journal of Endocrinology 2004150 681-686.

9 Lenders JW, Pacak K, Walther MM, Linehan WM, Mannelli M, Friberg P, Keiser HR, Goldstein DS \& Eisenhofer G. Biochemical diagnosis of pheochromocytoma: which test is best? Journal of the American Medical Association 2002287 1427-1434.

10 Plouin PF \& Gimenez-Roqueplo AP. Initial work-up and long-term follow-up in patients with phaeochromocytomas and paragangliomas. Best Practice \& Research. Clinical Endocrinology and Metabolism 200620 421-434.
11 Pacak K \& Eisenhofer G. An assessment of biochemical tests for the diagnosis of pheochromocytoma. Nature Clinical Practice. Endocrinology and Metabolism 20073 744-745.

12 Eisenhofer G, Goldstein DS, Walther MM, Friberg P, Lenders JW, Keiser HR \& Pacak K. Biochemical diagnosis of pheochromocytoma: how to distinguish true- from false-positive test results. Journal of Clinical Endocrinology and Metabolism $2003 \mathbf{8 8}$ 2656-2666.

13 Mannelli M, Ianni L, Cilotti A \& Conti A. Pheochromocytoma in Italy: a multicentric retrospective study. European Journal of Endocrinology 1999141 619-624.

14 Plouin PF, Degoulet P, Tugaye A, Ducrocq MB \& Menard J. Screening for phaeochromocytoma: in which hypertensive patients? A semiological study of 2585 patients, including 11 with phaeochromocytoma (author's transl) La Nouvelle Presse Médicale 198110 869-872.

15 Weismann D, Fassnacht M, Schubert B, Bonfig R, Tschammler A, Timm S, Hahner S, Wunder C \& Allolio B. A dangerous liaison pheochromocytoma in patients with malignant disease. Annals of Surgical Oncology 200613 1696-1701.

16 Crout JR \& Sjoerdsma A. Turnover and metabolism of catecholamines in patients with pheochromocytoma. Journal of Clinical Investigation $1964 \mathbf{4 3} 94-102$.

17 Feldman JM, Blalock JA, Zern RT \& Wells SA Jr. The relationship between enzyme activity and the catecholamine content and secretion of pheochromocytomas. Journal of Clinical Endocrinology and Metabolism 197949 445-451.

18 Motta-Ramirez GA, Remer EM, Herts BR, Gill IS \& Hamrahian AH. Comparison of CT findings in symptomatic and incidentally discovered pheochromocytomas. AJR. American Journal of Roentgenology 2005185 684-688.

19 Noshiro T, Shimizu K, Watanabe T, Akama H, Shibukawa S, Miura W, Ito S \& Miura Y. Changes in clinical features and longterm prognosis in patients with pheochromocytoma. American Journal of Hypertension 200013 35-43.

20 Stenstrom G \& Svardsudd K. Pheochromocytoma in Sweden 1958-1981. An analysis of the National Cancer Registry Data. Acta Medica Scandinavica $1986220225-232$.

21 Amar L, Servais A, Gimenez-Roqueplo AP, Zinzindohoue F, Chatellier G \& Plouin PF. Year of diagnosis, features at presentation, and risk of recurrence in patients with pheochromocytoma or secreting paraganglioma. Journal of Clinical Endocrinology and Metabolism 200590 2110-2116.

22 Herrera MF, Grant CS, van Heerden JA, Sheedy PF \& Ilstrup DM. Incidentally discovered adrenal tumors: an institutional perspective. Surgery $19911101014-1021$.

23 Hedeland H, Ostberg G \& Hokfelt B. On the prevalence of adrenocortical adenomas in an autopsy material in relation to hypertension and diabetes. Acta Medica Scandinavica $1968 \mathbf{1 8 4}$ 211-214.

24 Patocs A, Karadi E, Toth M, Varga I, Szucs N, Balogh K, Majnik J, Glaz E \& Racz K. Clinical and biochemical features of sporadic and hereditary phaeochromocytomas: an analysis of 41 cases investigated in a single endocrine centre. European Journal of Cancer Prevention 200413 403-409.

25 Mantero F, Terzolo M, Arnaldi G, Osella G, Masini AM, Ali A, Giovagnetti M, Opocher G \& Angeli A. A survey on adrenal incidentaloma in Italy. Study Group on Adrenal Tumors of the Italian Society of Endocrinology. Journal of Clinical Endocrinology and Metabolism 200085 637-644.

26 Lenert JT, Barnett CC Jr, Kudelka AP, Sellin RV, Gagel RF, Prieto VG, Skibber JM, Ross MI, Pisters PW, Curley SA, Evans DB \& Lee JE. Evaluation and surgical resection of adrenal masses in patients with a history of extra-adrenal malignancy. Surgery 2001130 1060-1067.

27 Adler JT, Mack E \& Chen H. Isolated adrenal mass in patients with a history of cancer: remember pheochromocytoma. Annals of Surgical Oncology $2007142358-2362$.

28 Zelinka T, Widimsky J \& Weisserova J. Diminished circadian blood pressure rhythm in patients with asymptomatic normotensive pheochromocytoma. Physiological Research 200150 631-634. 
29 Weismann D, Fassnacht M, Weinberger F, Hamelmann W, Diehl S, Lorenz K, Baerlehner E, Reincke M, Beuschlein F, Knoefel W, Nies C, Hahner S \& Allolio B. Intraoperative haemodynamic stability in patients with phaeochromocytoma - minimally invasive vs conventional open surgery. Clinical Endocrinology 200665 352-358.

30 Reisch N, Walz MK, Erlic Z \& Neumann HP. Pheochromocytoma still a challenge. Internist $2009 \mathbf{5 0} 27-35$.

31 Walz MK, Peitgen K, Saller B, Giebler RM, Lederbogen S, Nimtz K, Mann K \& Eigler FW. Subtotal adrenalectomy by the posterior retroperitoneoscopic approach. World Journal of Surgery 199822 621-627.
32 Lowel H, Meisinger C, Heier M, Hymer H, Alte D \& Volzke H. Epidemiology of hypertension in Germany. Selected results of population-representative cross-sectional studies. Deutsche Medizinische Wochenschrift 2006131 2586-2591.

Received 29 May 2009

Accepted 4 June 2009 